\section{Zur Bedeutung der Liquorbewegung}

Die beobachtete aktive Liquorströmung kann eine Beschleunigung der Lösungs-, Diffusions- und Verteilungsvorgänge im Liquor bewirken, ist also vergleichbar einem Rührwerk. Letzteres beleuchtet die noch wenig bekannte physiologisch-chemische Funktion des Liquors (vgl. F i s cher und $\mathrm{Ge} \mathrm{org} \mathrm{i}{ }^{2}$ u. a.). Hier wurde weniger die physiologische Funktion, als die physikalische Bedeutung einer Durchmischung in Betracht gezogen.

Worauf ich abschließend aber noch besonders hinweisen möchte, ist die Tatsache, daß die beobachteten Liquorströmungen an Amphibien-Larven gewonnen wurden, also in bestimmten Phasen der Individualentwicklung. Über die Funktionen des Liquors während der Embryonalentwicklung ist derzeit fast nichts bekannt.

\title{
Ein Beitrag zur Frage des Kalkstoffwechsels unserer Süßwasserschnecken
}

\author{
Von Ewald Frömming ${ }^{*}$ \\ (Z. Naturforschg. 8 b, 258-263 [1953]; eingegangen am 17. März 1953)
}

\begin{abstract}
Die herrschende Theorie, wonach unsere Süßwasserschnecken den zum Bau ihres Gehäuses notwendigen Kalk dem Wasser bzw. mineralischem Vorkommen entnehmen, wird abgelehnt. Es trifft nicht zu, daß nur in kalkhaltigem Wasser wohlausgebildete Gehäuse angetroffen werden, während umgekehrt in saurem Wasser die Schneckengehäuse stets kümmerlich, dünn und korrodiert sind und die dort lebenden Gastropoden sich ihren Kalk durch Benagen anderer Gehäuse beschaffen. Gehäuse-Korrosionen werden auch in kalkreichem Wasser beobachtet und sind in erster Linie auf Zerstörungen durch bohrende Algen zurückzuführen - die chemische Auflösung der Kalkschicht ist eine sekundäre Erscheinung.

Am Beispiel von Vertretern der Gattung Viviparus wird gezeigt, daß die Gehäusestärke genotypisch bedingt und nicht vom Kalkgehalt des Wohngewässers abhängig ist. Sofern in einem kalkreichen Gewässer Populationen mit statistisch realen starken Gehäusen auftreten, ist dies auf den höheren Kalkgehalt der Nahrungspflanzen zurückzuführen. Ausschlaggebend für das Gedeihen unserer limnischen Gastropoden ist nicht der Härtegrad oder die aktuelle Reaktion ihres Milieus, sondern das Vorhandensein geeigneter Nahrungspflanzen.
\end{abstract}

$\mathrm{S}_{\mathrm{C}}^{\mathrm{ei}}$ eit bekannt ist, daß die Süßwasserschnecken ihr Gehäuse aus kohlensaurem Kalk (Aragonit) aufbauen, besteht auch die Ansicht, daß die Tiere den dazu benötigten Kalk durch Benagen von geeignetem Gestein oder direkt aus dem umgebenden Wasser gewinnen - die Schnecken sind also an kalkreiches Wasser gebunden (wie diese Kalkgewinnung vor sich gehen soll, ist übrigens ein Geheimnis) und nur in kalkreichem Wasser entstehen wohlausgebildete, große Gehäuse. Umgekehrt aber heißt es: kalkarmes Wasser ist arten- und individuenarm, wo aber doch Schnecken vorkommen, handelt es sich um sehr dünnschalige Kümmerlinge; geraten Exemplare mit ausgebildetem Gehäuse in solches Wasser, werden diese korrodiert und schließlich zerstört. Auch fallen die Tiere über einander her und benagen eifrig die Gehäuse, ganz gleich ob diejenigen fremder Arten oder von Artgenossen, um so ihren Kalkhunger zu stillen, wie man es besonders in Aquarien beobachten kann.

Die scheinbar in die Augen springende Logik dieser Theorie (denn es ist nur eine Theorie, bisher noch

\footnotetext{
* Berlin N 20, Osloer Straße 99.
}

durch kein Experiment bewiesen) war so zwingend, daß noch keine gegenteilige Ansicht durchgedrungen ist. Gelegentliche Hinweise darauf, daß gerade in den kalkarmen Urgesteinsbächen die dickschaligsten $\mathrm{Mu}$ scheln gefunden werden, daß auch in kalkarmen Moorgewässern durchaus normal gebildete Molluskengehäuse vorkommen können und daß korrodierte Gehäuse auch in kalkreichem Wasser zu finden sind, wurden entweder ignoriert oder als $\mathrm{Zu}$ fälligkeiten abgetan.

Neuerdings hat nun $\mathrm{Hubendick}$ zu diesen Fragen Stellung genommen ${ }^{1}$ und kam zu dem $S c h l u ß$, ,daß der Kalk der wichtigste Milieufaktor für die limnische Gastropodenfauna “ ist und daß Kalkmangel der wesentlichste begrenzende Faktor für diese Fauna ist“. Weiter heißt es dann bei diesem Autor: „Daß die Kalkversorgung wirklich ein kritischer Faktor für die limnischen Gastropoden in kalkarmen Gebieten sein kann, habe ich durch Messungen der Schalendicke festgestellt." Ihm scheinen jedoch dann selbst Bedenken an der Beweiskraft dieser Methode gekommen zu sein, denn am Schluß des Absatzes heißt es einschränkend: „Daß die Schalenbildung in den kalkarmen Gebieten wirklich gehemmt ist, scheint also festzustehen." In bezug auf die Reaktion des Wassers hat 
$\mathrm{Hu}$ be ndick festgestellt, daß die Artenfrequenz eine deutliche Korrelation mit dem $p_{\mathrm{H}}$-Wert zeigt.

Der schwedische Autor hat viel Material zusammengetragen, es betrifft jedoch lediglich die Süßwasserschnecken Südschwedens; in diesem Landstrich liegen aber ganz andere Verhältnisse vor als in Mitteleuropa, und schon aus diesem Grunde wäre es richtiger gewesen, wenn $\mathrm{Hubendick}$ seine Ergebnisse nicht verallgemeinert hätte. Es fällt auch auf, daß der Autor für seine Theorie voreingenommen ist: so teilt er mit Boycott die limnischen Gastropoden nach ihrem Kalkbedarf in drei Gruppen ein und fand, daß 9 Arten Kalkarmut vertragen, 12 Arten einen geringen Kalkbedarf haben und nur 5 Arten „deutlich kalkfordernd“ sind - unter den letzteren befinden sich auch Spiralina vortex und Segmentina nitida! Letztere ist vom Kontinent aus Wiesensümpfen, Gräben, Moorgewässern bekannt und darf beinahe als Charakterschnecke kalkarmer, wo nicht saurer, Gewässer angesprochen werden. Diese Aufstellung von $\mathrm{Huben-}$ di c k spricht also gegen seine eigene Ansicht. Erwähnt sei auch, daß H u be n d i c k mir vorwirft, ich hätte Viviparus viviparus und Viviparus fasciatus ,verwechselt“ (als ob man diese größte europäische Süßwasserschnecke überhaupt mit der verwandten Art verwechseln könnte, noch dazu, wennn man dreißig Jahre mit diesen Tieren gearbeitet hat); tatsächlich ist es so, daß in England und den ihm folgenden Ländern diese beiden Arten gerade umgekehrt als auf dem Kontinent bezeichnet werden was Herrn $\mathrm{H} \mathrm{u}$ be nd i ck bekannt sein müßte 2.

Wenn man nun die in der Literatur niedergelegten Behauptungen unvoreingenommen prüft, kommt man zu ganz anderen Schlußfolgerungen. Da ist zunächst festzustellen, daß von den älteren Malakologen keinerlei chemische Prüfung eines Gewässer vorgenommen wurde. Ich habe nun in jahrelangen Wanderungen die brandenburgischen, pommerschen ${ }^{3}$, neumärkischen, mecklenburgischen ${ }^{4}$, oldenburgischen und niederländischen (1952) Gewässer der verschiedensten Typen untersucht und dabei keine Stütze für die geltende Anschauung finden können. Bei eingehenden Aquariumversuchen ${ }^{5,6}$ verhielt es sich ebenso. Unsere häufigeren, einheimischen Süßwasser-

1 B. H u b e n d i c k, Die Verbreitungsverhältnisse der limnischen Gastropoden in Südschweden. Zool. Bidr. fran Uppsala 24, 419-559 [1947].

$2 \mathrm{Hub}$ endick zitiert auch ungenau; so sagt er auf S. 481/82 von Viviparus viviparus: „In der deutschen Literatur wird oft angegeben, daß sie hauptsächlich an fließendes Wasser gebunden sei." Mir ist nicht eine Stelle in deutschen Schriften bekannt, wo Derartiges behauptet wird. $\mathrm{Hubendick}$ meint natürlich V.fasciatus, also ist ihm die verschiedenartige Benennung der Arten bekannt (wie auch aus S. 458 hervorgeht).

3 E. F r ö m m in g, Über den Einfluß der Wasserstoffionenkonzentration auf unsere Süßwasserschnecken. Intern. Rev. ges. Hhydrobiol. 33, 25-37 [1936].

4 E. Frö m ming, Untersuchungen über den Einfluß der Härte des Wohngewässers auf das Vorkommen unserer Süßwassermollusken. Intern. Rev. ges. Hydrobiol. 36, 531—561 [1938]. schnecken kommen in den Gewässern mit allen Härtegraden und den verschiedensten Reaktionen vor. Das Gebundensein einer Art an einen bestimmten Härtegrad oder an einen bestimmten $p_{\mathrm{H}}$-Bereich war nicht festzustellen. Schwankungen in der Populationsdichte und in der Artenanzahl kommen natürlich vor - sie können aber den Gesamteindruck nicht verwischen und sind durch andere Faktoren, z. B. Wasserbewegung, bedingt.

In Torflöchern, Moorgewässern, schattenreichen, sauren Waldweihern usw. kann häufig eine Artenund Individuenarmut beobachtet werden, und zwar um so sicherer, als das bräunliche, klare Wasser einen Blick bis auf den Grund ermöglicht - kaum jemals wird das Auge durch Pflanzenwuchs daran gehindert. Dies aber ist der eigentliche Grund für die Schneckenarmut, dies ist auch der Grund, warum die vorhandenen Tiere so kümmerlich wirken und so dünne $\mathrm{Ge}$ häuse haben. Diese Tiere leben in einem chronischen Hungerzustand, da es ihnen an Nahrung, eben den höheren Pflanzen, gebricht. Sie bilden nämlich in lebensfrischem oder abgestorbenem Zustand die Nahrung sehr vieler unserer limnischen Gastropoden. Mit ihnen nehmen sie auch den zum Aufbau des Gehäuses benötigten Kalk auf. Fehlt nun diese Nahrung, so müssen die Tiere kümmern und können auch kein normales Gehäuse bauen - mit dem Kalkgehalt des Wassers, seiner aktuellen Reaktion oder dem Gehalt an Huminsäuren aber hat das nichts zu tun ${ }^{7}$.

Hier sei noch einmal $\mathrm{H} \mathrm{u}$ b e $\mathrm{n} \mathrm{d} \mathrm{i} \mathrm{c} \mathrm{k} \mathrm{zitiert,} \mathrm{welcher} \mathrm{der}$ Meinung ist, daß „die extrem sauren, dystrophen Gewässer durchaus imstande sind, dem höchst undifferenzierten Nahrungsbedarf der Gastropoden zu genügen.“ Auch hier kann ich Herrn $\mathrm{H}$ u be n d i ck nicht beipflichten; jeder, der sich eingehend mit der Aufzucht verschiedener Schneckenarten beschäftigt hat (und das hält auch $\mathrm{Hu}$ be ndick auf S. 500 seiner Arbeit für ein wichtiges Kriterium), wird wissen, daß der Nahrungsbedarf unserer Gastropoden keineswegs "höchst undifferenziert" ist ${ }^{8}$.

5 E. Frömming, Untersuchungen über den Chemismus des Aquarienwassers. Intern. Rev. ges. Hydrobiol. 40, 361-367 [1941].

6 E. Frö m ming, Physa acuta Drap. in Berlin eingebürgert. Arch. Hydrobiol. 43, 304316 [1950].

7 Ähnlich hat sich auch W. A d e n s a m e r (SüßwasserMollusken Zentraleuropas, 3. Gattung Limnaea, Bl. Aq.Terr. Kde. 49, 9-11, 28-30 u. 44-46 [1938]) ausgedrückt: „Die Schnecken nehmen den zu ihrem Schalenbau nötigen Kalk durch ihre Nahrung auf. Der höhere oder geringere Kalkgehalt des Wohnwassers scheint mir nur indirekt oder überhaupt nicht ausschlaggebend zu sein.“

8 Auch Ehrmann (Leipzig 1933) wußte um die Nahrungsarmut in sauren Gewässern und sagt z. B. ausdrücklich von Radix peregra Müll., daß sie infolge „ihrer Anspruchslosigkeit in der Ernährung mehr als andere Wasserschnecken auch Bewohnerin der Moore und der höheren Gebirgslagen“" ist. 


\begin{tabular}{|c|c|c|c|c|c|c|}
\hline \multirow{2}{*}{ Fundort } & \multicolumn{3}{|c|}{ Viviparus viviparus $L}$. & \multicolumn{3}{|c|}{ Viviparus fasciatus Müll. } \\
\hline & Höhe & Breite & Gewicht & Höhe & Breite & Gewicht \\
\hline \multirow[t]{2}{*}{ Tegeler See bei Berlin } & $\begin{array}{l}25,7 \mathrm{~mm} \\
26,6 \mathrm{~mm} \\
27,3 \mathrm{~mm} \\
27,6 \mathrm{~mm} \\
28,4 \mathrm{~mm} \\
28,8 \mathrm{~mm} \\
31,3 \mathrm{~mm} \\
31,8 \mathrm{~mm}\end{array}$ & $\begin{array}{l}21,8 \mathrm{~mm} \\
21,9 \mathrm{~mm} \\
22,0 \mathrm{~mm} \\
21,5 \mathrm{~mm} \\
20,3 \mathrm{~mm} \\
22,4 \mathrm{~mm} \\
24,0 \mathrm{~mm} \\
25,1 \mathrm{~mm}\end{array}$ & $\begin{array}{l}0,68 \mathrm{~g} \\
0,92 \mathrm{~g} \\
0,86 \mathrm{~g} \\
0,64 \mathrm{~g} \\
0,92 \mathrm{~g} \\
0,98 \mathrm{~g} \\
1,16 \mathrm{~g} \\
1,04 \mathrm{~g}\end{array}$ & $\begin{array}{l}25,7 \mathrm{~mm} \\
26,8 \mathrm{~mm} \\
27,0 \mathrm{~mm} \\
27,2 \mathrm{~mm} \\
28,2 \mathrm{~mm} \\
29,0 \mathrm{~mm} \\
31,3 \mathrm{~mm} \\
31,7 \mathrm{~mm}\end{array}$ & $\begin{array}{l}19,5 \mathrm{~mm} \\
19,7 \mathrm{~mm} \\
20,8 \mathrm{~mm} \\
20,8 \mathrm{~mm} \\
20,2 \mathrm{~mm} \\
20,8 \mathrm{~mm} \\
22,8 \mathrm{~mm} \\
22,9 \mathrm{~mm}\end{array}$ & $\begin{array}{l}1,19 \mathrm{~g} \\
1,15 \mathrm{~g} \\
1,12 \mathrm{~g} \\
1,14 \mathrm{~g} \\
1,26 \mathrm{~g} \\
1,48 \mathrm{~g} \\
1,70 \mathrm{~g} \\
1,78 \mathrm{~g}\end{array}$ \\
\hline & D. : $28,4 \mathrm{~mm}$ & $22,4 \mathrm{~mm}$ & $0,90 \mathrm{~g}$ & $28,3 \mathrm{~mm}$ & $20,9 \mathrm{~mm}$ & $1,35 \mathrm{~g}$ \\
\hline \multirow[t]{2}{*}{$\begin{array}{l}\text { Lehnitz-See } \\
\text { bei Oranienburg }\end{array}$} & $\begin{array}{l}25,2 \mathrm{~mm} \\
25,4 \mathrm{~mm} \\
26,3 \mathrm{~mm} \\
26,5 \mathrm{~mm} \\
27,2 \mathrm{~mm} \\
30,8 \mathrm{~mm} \\
31,2 \mathrm{~mm} \\
33,7 \mathrm{~mm} \\
34,8 \mathrm{~mm}\end{array}$ & $\begin{array}{l}19,5 \mathrm{~mm} \\
19,9 \mathrm{~mm} \\
20,9 \mathrm{~mm} \\
21,8 \mathrm{~mm} \\
22,7 \mathrm{~mm} \\
23,0 \mathrm{~mm} \\
24,4 \mathrm{~mm} \\
27,0 \mathrm{~mm} \\
26,7 \mathrm{~mm}\end{array}$ & $\begin{array}{l}0,58 \mathrm{~g} \\
0,67 \mathrm{~g} \\
0,74 \mathrm{~g} \\
0,96 \mathrm{~g} \\
0,87 \mathrm{~g} \\
1,00 \mathrm{~g} \\
1,24 \mathrm{~g} \\
1,48 \mathrm{~g} \\
1,55 \mathrm{~g}\end{array}$ & $\begin{array}{l}25,8 \mathrm{~mm} \\
26,0 \mathrm{~mm} \\
26,3 \mathrm{~mm} \\
27,2 \mathrm{~mm} \\
27,2 \mathrm{~mm} \\
30,0 \mathrm{~mm} \\
30,3 \mathrm{~mm} \\
30,6 \mathrm{~mm} \\
31,1 \mathrm{~mm}\end{array}$ & $\begin{array}{l}19,4 \mathrm{~mm} \\
20,0 \mathrm{~mm} \\
19,7 \mathrm{~mm} \\
20,0 \mathrm{~mm} \\
20,4 \mathrm{~mm} \\
21,8 \mathrm{~mm} \\
21,8 \mathrm{~mm} \\
21,8 \mathrm{~mm} \\
22,2 \mathrm{~mm}\end{array}$ & $\begin{array}{l}1,07 \mathrm{~g} \\
1,18 \mathrm{~g} \\
1,39 \mathrm{~g} \\
1,37 \mathrm{~g} \\
1,41 \mathrm{~g} \\
1,80 \mathrm{~g} \\
1,82 \mathrm{~g} \\
1,79 \mathrm{~g} \\
1,76 \mathrm{~g}\end{array}$ \\
\hline & D. : $29,0 \mathrm{~mm}$ & $22,9 \mathrm{~mm}$ & $1,01 \mathrm{~g}$ & $28,3 \mathrm{~mm}$ & $20,8 \mathrm{~mm}$ & $1,51 \mathrm{~g}$ \\
\hline \multirow[t]{2}{*}{$\begin{array}{l}\text { Neuer Hafen } \\
\text { in Deventer (Niederlande) }\end{array}$} & $\begin{array}{l}28,0 \mathrm{~mm} \\
29,8 \mathrm{~mm} \\
29,9 \mathrm{~mm} \\
31,4 \mathrm{~mm} \\
31,6 \mathrm{~mm} \\
31,8 \mathrm{~mm} \\
33,3 \mathrm{~mm} \\
35,2 \mathrm{~mm}\end{array}$ & $\begin{array}{l}22,4 \mathrm{~mm} \\
23,8 \mathrm{~mm} \\
24,7 \mathrm{~mm} \\
26,2 \mathrm{~mm} \\
24,9 \mathrm{~mm} \\
26,0 \mathrm{~mm} \\
26,6 \mathrm{~mm} \\
27,3 \mathrm{~mm}\end{array}$ & $\begin{array}{l}0,67 \mathrm{~g} \\
1,02 \mathrm{~g} \\
1,06 \mathrm{~g} \\
0,97 \mathrm{~g} \\
1,26 \mathrm{~g} \\
1,30 \mathrm{~g} \\
1,94 \mathrm{~g} \\
2,08 \mathrm{~g}\end{array}$ & $\begin{array}{l}27,3 \mathrm{~mm} \\
28,6 \mathrm{~mm} \\
29,3 \mathrm{~mm} \\
31,5 \mathrm{~mm} \\
32,2 \mathrm{~mm} \\
33,2 \mathrm{~mm} \\
34,8 \mathrm{~mm} \\
35,7 \mathrm{~mm}\end{array}$ & $\begin{array}{l}21,2 \mathrm{~mm} \\
20,1 \mathrm{~mm} \\
22,6 \mathrm{~mm} \\
23,8 \mathrm{~mm} \\
23,2 \mathrm{~mm} \\
24,2 \mathrm{~mm} \\
24,8 \mathrm{~mm} \\
25,8 \mathrm{~mm}\end{array}$ & $\begin{array}{l}1,24 \mathrm{~g} \\
1,30 \mathrm{~g} \\
1,54 \mathrm{~g} \\
1,85 \mathrm{~g} \\
2,05 \mathrm{~g} \\
1,87 \mathrm{~g} \\
2,36 \mathrm{~g} \\
2,53 \mathrm{~g}\end{array}$ \\
\hline & D. $: 31,4 \mathrm{~mm}$ & $25,2 \mathrm{~mm}$ & $1,29 \mathrm{~g}$ & $31,6 \mathrm{~mm}$ & $23,2 \mathrm{~mm}$ & $1,84 \mathrm{~g}$ \\
\hline
\end{tabular}

Tab. 1. Übersicht über die Gehäuse-Gewichte zweier Viviparus-Arten von verschiedenen Fundorten.

Was nun die Stärke der Gehäusewandung im besonderen angeht, so ist darüber schon viel geschrieben worden; ich beschränke mich aber darauf, hier einige Beispiele aus eigenem Material anzuführen. Sie betreffen die beiden bereits erwähnten Arten der Gattung Viviparus, die in demselben Wohngewässer an derselben Stelle eingesammelt wurden. Aus vielen Exemplaren habe ich die ungefähr gleichgroßen Stücke herausgesucht, gemessen, gewogen und die Zahlen in der Tabelle zusammengestellt. Ein Blick auf die Tabelle zeigt deutlich die Unterschiede: das Gehäuse etwa gleichgroßer (dabei wesentlich schmälerer) Viviparus fasciatus ist immer um $20-30 \%$ schwerer als das der verwandten Art. Da die Tiere eines Gewässers stets von derselben Stelle stammen, ist es unmöglich, daß der Kalkgehalt des Milieus oder die Reaktion desselben hierfür verantwortlich gemacht werden können und es bleibt der alleinige Schluß, daß die Dickschaligkeit des V.fasciatus-Gehäuses genotypisch bedingt ist. Schon $\mathrm{F} \mathrm{r} \mathrm{a} \mathrm{n}{ }^{9}$ hat übrigens darauf hingewiesen. $\mathrm{Ob}$ sich in dieser Hinsicht alle Schneckenarten gleich verhalten, muß noch untersucht werden, und diese Untersuchungen wären wichtiger als theoretische Erörterungen. Ich glaube nicht, daß Viviparus fasciatus Müll. eine Ausnahme von der allgemeinen Regel darstellt, sondern bin der Ansicht, daß jede Schneckenart ihr Gehäuse nach artgemäßem Charakter aufbaut - sowohl in der Form wie in der Stärke und Färbung. Je nachdem, ob die Tiere in einem optimalen oder in einem unzureichenden Milieu leben, wird das Gehäuse natürlich von dem spezifischen Artcharakter Abweichungen zeigen. Das Optimum eines Milieus setzt sich nun aus verschiedenen Faktoren zusammen, in erster Linie ist dafür aber unbedingt der Reichtum an verwendbaren Nahrungs-

9 V.Franz, Über Bastardpopulationen in der Gattung Paludina (recte: Viviparus). Biol. Zbl. 48, 79-93 [1928]. 
stoffen anzusehen! Das Tier ist jedoch nicht nur einfach ein Konsument der Pflanzen, sondern seine Konstitution, seine Vermehrungsfreudigkeit, die Größe der Eier und nicht zuletzt die von den Drüsen ausgeschiedenen Baustoffe für das Gehäuse sind weitgehend von der Zuführung ausreichender Nahrungsmengen abhängig. Der Kalkgehalt des Wohngewässers aber ist allenfalls von mittelbarer Bedeutung, indem nämlich Pflanzen, die in kalkreichem Wasser vegetieren, mehr Kalk enthalten als andere — ob dies in der Tat immer so ist, entzieht sich meiner Kenntnis. In jedem Falle ist aber für das Gedeihen einer Art der Nahrung das Primat zuzuerkennen. Dies ist keine spekulative Behauptung, sondern durch den exakten biologischen Versuch nachzuweisen ${ }^{10}$.

Wie steht es nun mit den Korrosionen? Wie eine genauere Betrachtung der betroffenen Gehäuse ergibt, gehen diese Zerfressungen stets von Kratzern und Schrammen aus, sofern nicht ganze Partien des Periostrakums abgeschürft sind. Diese Kratzer entstehen, wenn die Tiere über kantige Steine kriechen, verholzte Pflanzenteile streifen usw. Besonders häufig treten sie naturgemäß in einem mit Steinen versehenen Aquarium auf, wo die Tiere nur eine sehr beschränkte Bewegungsmöglichkeit haben und immer wieder denselben Weg kriechen müssen. Bei diesem Entlangstreifen an einer Steinecke entsteht auf dem Gehäuse ein Kratzer und gewöhnlich ist hier das Periostrakum durchschnitten worden. Jetzt liegt die Kalkschicht frei und ist äußeren Einwirkungen zugänglich - eine Wirkung der im Wasser vorhandenen freien Kohlensäure auf das intakte Gehäuse einer lebenden Schnecke halte ich für ausgeschlossen, mag das Wasser so sauer sein wie es will! Von solchen Korrosionen findet man in freien Gewässern besonders die Viviparus-, Theoduxus ${ }^{11}$ und BithyniaGehäuse entstellt, da diese Arten gern zwischen den Granitbrocken leben, die zur Befestigung künstlicher Wasserstraßen am Ufer geschichtet sind. Auch an gemauerten Kanalufern kann man diese Erscheinung beobachten, solange diese nicht mit dicken Algenpolstern bedeckt sind; hier findet allerdings mehr ein Abschürfen des Periostrakums an jenen Stellen statt, an denen das Gehäuse auf den Steinen schleift. Es ist dies häufig der Apex. Dieser älteste und oft am mei-

10 E. Frömming, Ein Ernährungsversuch mit der Süßwasserlungenschnecke Physa acuta Drap. Z. Naturforschg. 2 b, 419—421 [1947].

11 E. Frömming, Über das Gehäuse von Theodoxus fluviatilis L. Intern. Rev. ges. Hydrobiol. 33, 305-312 [1936]. sten exponierte Teil des Gehäuses ist Verletzungen und Abschürfungen in erster Linie ausgesetzt, somit der freien Kohlensäure den Angriff ermöglichend.

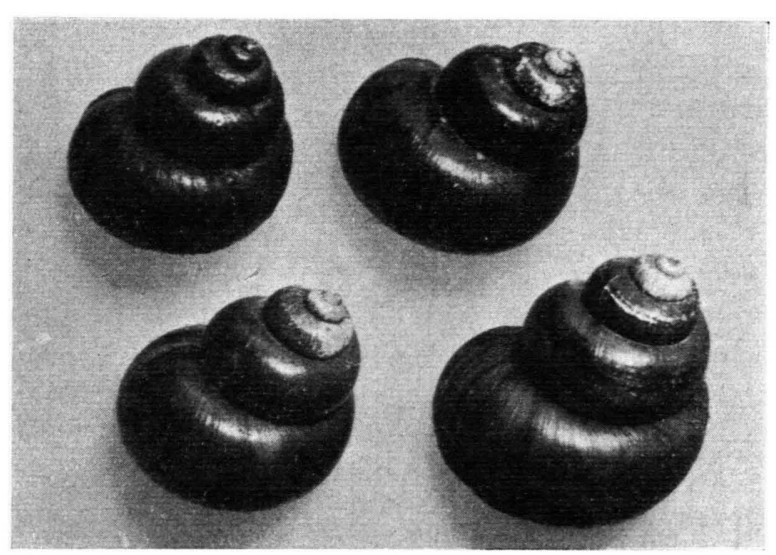

Abb. 1. V.viviparus aus dem Tegeler See bei Berlin, alle Gehäuse von derselben Stelle. Neben einem normalen Gehäuse die übrigen mit Korrosionen verschiedenen Grades am apicalen Teil. Auf dem dritten Umgang des größten Gehäuses sieht man deutlich die gekratzten Radialstreifen, die entstehen, wenn das Tier durch eine Welle auf dem Seegrund herumgerollt wird und dabei einen kantigen Stein streift. (Phot. M. R. Z a h n.)

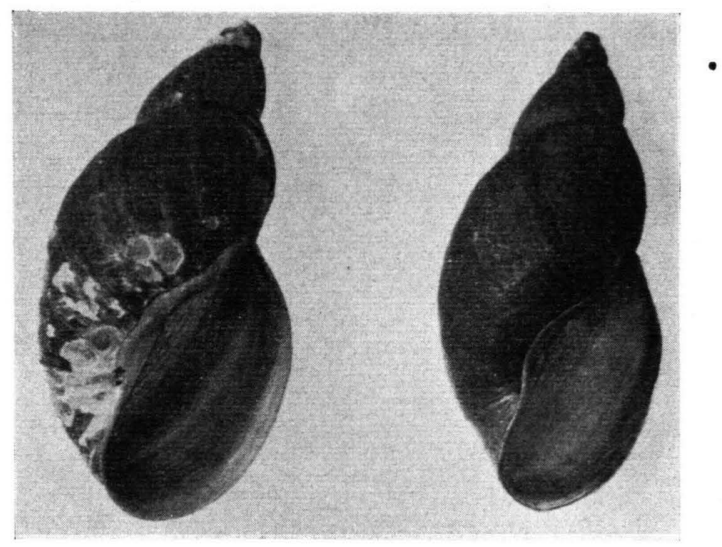

Abb. 2. Gehäuse von Galba palustris aus einem Dorfteich in Bergfelde bei Berlin; neben einem normalen ein stark korrodiertes, bei dem manche kraterartigen Vertiefungen die Gehäusewand nahezu durchbohrt haben. Beide Tiere wurden an derselben Stelle gefangen und sind offensichtlich gleich alt. (Phot. M. R. Z a h n.)

Auch die an Seeufern, welche mit grobem Sand oder Steinen bedeckt sind, lebenden Schlammschnecken und Planorbiden zeigen nicht selten solche Verletzungen ihrer Gehäuse. Bei den Tellerschnecken liegen die empfindlichen Embryonalwindungen allerdings 
in der Mitte des Gehäuses, und von B rand $t^{12}$ ist der Ansicht, daß hier (wie überhaupt) die Korrosion durch gegenseitiges Benagen entsteht. Ich halte es aber für höchst unwahrscheinlich, daß sich die Schnecken gerade immer die Mitte des Gehäuses für ihren Angriff aussuchen und bin der Ansicht, daß an diesen ältesten Windungen das Konchiolin spröde wird, Risse bekommt und dann Angriffspunkte bietet.

Neben der chemischen Entkalkung des Molluskengehäuses (Auflösung der frei liegenden Aragonitschicht durch agressive Kohlensäure), der ich nur eine beschränkte, lokale Verbreitung zuerkenne, ist die durch das Eindringen von pflanzlichen Organismen hervorgerufene ungleich wichtiger und weltweit verbreitet. Die befallenen Gehäuse zeigen grubige Vertiefungen, welche schließlich die Gehäusewand glatt durchlöchern können, kraterartige Mulden, minenartige Gänge usw. Diese Zerstörungen werden durch Algen verschiedener Art hervorgerufen, welche sich in der verletzten Konchinschicht angesiedelt haben und nicht nur durch ihr Eindringen in das Substrat, sondern auch durch ihren Atmungs-Stoffwechsel (Kohlensäure-Ausscheidung) eine Entkalkung der befallenen Stellen verursachen; hier wirkt also die mechanische und die chemische Zerstörung zusammen. Hier findet sich auch die durchaus zwanglose Erklärung dafür, daß so häufig Korrosionen von Molluskenschalen in kalkreichem Wasser beobachtet werden ${ }^{13}$, worauf ich bereits früher ${ }^{4}$ hingewiesen habe und was $\operatorname{Roth} \mathrm{h}^{14}$ schon vor 40 Jahren sagte. Übrigens müßten ja, wenn das saure Waser an sich die Korrosionen hervorruft, sämtliche Gehäuse der in solchem Wasser lebenden Arten und alle Individuen einer Art davon betroffen sein - das ist aber nie der Fall. Neben Gehäusen mit stark zerstörter Oberfläche findet man immer auch solche (gleicher Größe!), die völlig unbeschädigt sind.

12 A.v. Brandt, Kohlensäureschäden an Mollusken in kleinen sauren Waldgewässern. Arch. Moll. 68, 120-127 [1936].

13 Auch hier nur ein Beispiel aus der neueren Literatur: K. Regius traf in einem Teich bei Magdeburg auf ein Massenvorkommen von z. Tl. recht großen Bithynia tentaculata L. Es handelte sich um alkalisches Wasser mit einer Gesamthärte von $18,7^{\circ}(166 \mathrm{mg} / l \mathrm{CaO})$. Viele Gehäuse waren korrodiert, besonders zeigten sich die bekannten Radialstreifen; R e g i u s hielt diese „Gehäuseanfressungen (vermutlich durch Artgenossen hervorgerufen) " und wunderte sich sehr über deren Regelmäßigkeit. - In diesem Gewässer fanden sich übrigens „,zahlreich dünnschalige“ Lymnaea stagnalis! Individuelle Variabilität bei Bithynia tentaculata Linné. Abh. Mus. Naturk. Magdeburg 7, 183-197 [1939].

14 W. R oth, Die Korrosion der Schale bei unseren Süßwasserschnecken. Bl. Aq.-Terr. Kde. 21, H. 6-8 [1910].
Pe ye ${ }^{15}$ hat am Beispiel von Nerita, Neritina und Ampullaria gezeigt, daß ,an der korrodierten apikalen Partie zahlreiche bohrende pflanzliche Organismen in die Schale eindringen". Durch instruktive Mikrofotos von Dünnschliffen erhärtet P e y e r seine interessanten Ausführungen. Wenn er aber die Vermutung ausspricht, daß „die primäre Ursache der Zerstörung der Konchinschicht in dem Eindringen von bohrenden pflanzlichen Organismen liegen könnte", so vermag ich ihm nicht zu folgen. Die unversehrte Konchinschicht ist meiner Überzeugung nach weder für pflanzliche Organismen noch für die freie Kohlensäure angreifbar - erst die mechanische Verletzung, mag sie noch so gering sein, schafft die Möglichkeit hierfür; die Embryonalwindungen als der älteste und schwächste Teil eines Schneckengehäuses sind allerdings solchen Beschädigungen in erster Linie ausgesetzt.

Zum Schluß noch ein Wort über die Gehäuseanfressungen durch Schnecken ${ }^{16}$. Wo in Aquarien dergleichen beobachtet wird, ist es unbedingt eine durch unzweckmäßige Haltung hervorgerufene Anomalie. In den meisten Fällen wird es sich aber meiner Meinung nach um eine ungenaue Beobachtung bzw. eine falsche Schlußfolgerung handeln. Wenn eine Schnecke stirbt, sinkt sie zu Boden, und schon kurze Zeit später sammeln sich die anderen Schnecken um den Kadaver und beginnen eifrig zu fressen. Da unsere Limnaeaen, Planorbiden und Physiden, wie ich nachgewiesen habe, tierisches Eiweiß zu ihrem Gedeihen brauchen, ihnen aber in einem Aquarium diese Stoffe gewöhnlich nur in unzureichender Menge geboten werden, stürzen sie sich sofort auf jeden Kadaver und verzehren ihn restlos. Schon nach 1 oder 2 Tagen findet man dann das sauber präparierte Ge-

15 B. P e y e r, Über Algen und Pilze in tierischen Hartsubstanzen. Arch. J. Klaus-Stiftg., Erg.-Bd. zu 20 , $495-546$ [1945].

16 Ein eifriger Verfechter dieser Ansicht ist auch heute noch $\mathrm{S}$ ch e r m e r (Hydrobiologische Untersuchungen im Travegebiet. Forschg. geogr. Ges. usw., 2. Reihe, H. 42, 35-131 [1950]; H. 43, 7-89 [1951]; er sagt z. B. von Stagnicola palustris Müll.: „Die größte Form fa. corvus Gmel. fällt oft durch zerfressene Gehäuse auf. Die Tiere fressen gegenseitig ihre Gehäuse an und die Humussäure zerstört sie weiter. Auch gewisse Algen von blaugrüner Farbe, die auf den Gehäusen ganze Büschel bilden, scheinen die Kalkschicht anzugreifen." Und von der Deckelsumpfschnecke heißt es in derselben Arbeit: „In Gräben der Torfmoore leidet Viviparus viviparus L. stark durch den Einfluß der Humussäure, die die Gehäuse zerfrißt, so daß oft die Spitze fehlt." Die S c h e r m e r sche Arbeit enthält auch sonst noch mancherlei Unrichtigkeiten, insbesondere über die Ernährung der Schnecken, doch gehe ich darauf a. O. ein. 
häuse des gestorbenen Tieres. Seit mehreren Jahrzehnten halte ich ständig Wasserschnecken im Aquarium, und noch niemals habe ich beobachtet, daß das Gehäuse einer gestorbenen (oder gar lebenden) Schnecke von den Artgenossen oder anderen Arten benagt worden wäre. In einem normal eingerichteten Aquarium kommt das auch anderswo nicht vor und ich bin der festen Úberzeugung, daß die betr. Beobachter nur sahen, wie die Schnecken an dem Kadaver saßen, und nun annahmen (befangen durch die herrschende Anschauung), sie nagten an dem Gehäuse, um ihren Kalkhunger zu stillen. Tatsächlich aber fraßen sie nur an dem Kadaver und ließen das Gehäuse unangetastet.

\title{
Die Abhängigkeit der Reizschwelle (Rheobase) isolierter Ranvierscher Schnürringe von der Ionenkonzentration
}

\author{
Von Hanschristoph LÜtTGaU * \\ Aus dem Zoologischen Institut der Universität Göttingen \\ (Z. Naturforschg. 8 b, 263-268 [1953]; eingegangen am 26. Februar 1953)
}

\begin{abstract}
Es wird die Reizschwelle isolierter Schnürringe motorischer Nervenfasern in Abhängigkeit von der Konzentration an K-, Na- und Ca-Ionen gemessen.
\end{abstract}

$\mathrm{I}$ m Jahre 1890 berichtete W. O st w a ld ${ }^{1}$ über die Entstehung von Potentialdifferenzen an semipermeablen Membranen und sprach die Vermutung aus, daß die elektrischen Ströme in Muskeln und Nerven durch die Eigenschaften solcher Membranen erklärt werden könnten. Es folgte bald darauf die Membrantheorie von Bernstein ${ }^{2}$. Elektrophysiologische und chemisch-physiologische Untersuchungen zeigten in den folgenden Jahren, daß die Plasmamembran nicht einfach einer physikalisch-chemischen Niederschlagsmembran gleichgesetzt werden kann, sondern daß es sich um eine „lebende“ Struktur handelt, an der sich komplizierte physiologische Vorgänge abspielen.

Wurden die Untersuchungen zuerst am ganzen Nervenstamm durchgeführt, so gelang es einigen Forschern ${ }^{3-5}$, Messungen an einzelnen isolierten Nervenfasern durchzuführen. „Der Fortschritt, der hier auf dem Gebiet der Nervenphysiologie erzielt wurde, ist vergleichbar mit dem Fortschritt in der Chemie eines Stoffgemisches, wenn es gelingt, aus dem Gemisch die einzelnen Komponenten als Kristalle

* Herrn Prof. Dr. H. A u tru m danke ich für die Anregung und das Interesse bei der Durchführung der Versuche.

1 W. Os t w a ld, Z. physik. Chem. 6, 71 [1890].

2 J. B ernstein, Pflügers Arch. ges. Physiol. Menschen Tiere 92, 521 [1902]; Elektrobiologie, Braunschweig [1912].

3 G. K a t o, Mikrophysiology of nerve, Tokio [1934].

4 I. T a s a k i, Pflügers Arrch. ges. Physiol. Menschen Tiere 244, 125 [1940]; Jap. J. Physiol. 1, 75 [1950]. $\mathrm{zu}$ isolieren "." Auf Grund von Einzelfaseruntersuchungen erwiesen sich die Vorstellungen Bernste in s, nach denen das Aktionspotential durch $\mathrm{Zu}$ sammenbruch des Ruhepotentials entsteht, als zu einfach. Deshalb wurde von $\mathrm{Hodgkin}, \mathrm{Huxley}$ und $\mathrm{Katz}^{7}$ eine erweiterte Membrantheorie entwickelt. Dabei bleiben jedoch viele Probleme ungelöst, insbesondere ist eine substantielle Unterbauung der Theorie noch nicht möglich ${ }^{8}$, und der Vorgang der Erregungsauslösung bleibt weiterhin mit vielen Rätseln behaftet ${ }^{9}$.

Weiterführen kann hier eine intensive Untersuchung der physiologischen Leistungen der Membran (Rheobase, Akkomodation, Ruhe- und Aktionspotential usw.) unter verschiedenen Bedingungen. Diesem Zweck soll auch die vorliegende Arbeit dienen. Im ersten Teil werden die Schwellen einzelner Ranvierscher Knoten bei verschiedenen Ionenkonzentrationen gemessen. Ein zweiter Teil über die Wirkungen von Faserdurchtrennung, katelektrotonischer Polarisation und KCl-Zusatz auf die Schwelle einzelner Schnürringe wird folgen.

5 H. J. Curtis u. K. S. Cole, J. cellular comparat. Physiol. 19, 135 [1942].

6 A. von Muralt, Die Signalübermittlung im Nerven, Basel [1946].

7 A. L. Hodgkin, A. F. Huxley u. B. Katz, Arch. Sci. de Physiol. 3, 129 [1949].

8 H. N e t t e r, Biologische Physikochemie, Akad. Verlagsgesellsch. Potsdam 1951.

9 A. L. Hodgkin, J. of Physiol. 106, 319 [1947]. 